\title{
Erratum to: Intercomparison of the Arctic sea ice cover in global ocean-sea ice reanalyses from the ORA-IP project
}

\author{
Matthieu Chevallier ${ }^{1}$ - Gregory C. Smith ${ }^{2}$ Frédéric Dupont ${ }^{3}$. \\ Jean-François Lemieux ${ }^{2}$ Gael Forget ${ }^{4} \cdot$ Yosuke Fujii $^{5} \cdot$ Fabrice Hernandez $^{6,7}$. \\ Rym Msadek $^{8,9}$ - K. Andrew Peterson ${ }^{10}$ - Andrea Storto ${ }^{11}$ - Takahiro Toyoda ${ }^{5}$. \\ Maria Valdivieso $^{12}$ - Guillaume Vernieres ${ }^{13,14}$ - Hao Zuo ${ }^{15}$ - Magdalena Balmaseda ${ }^{15}$. \\ You-Soon Chang ${ }^{16}$ - Nicolas Ferry ${ }^{6}$ - Gilles Garric ${ }^{6}$ - Keith Haines ${ }^{12} \cdot$ Sarah Keeley $^{15}$. \\ Robin M. Kovach $^{14} \cdot$ Tsurane Kuragano $^{5}$ - Simona Masina ${ }^{11,17}$ - Yongming Tang ${ }^{10,15}$. \\ Hiroyuki Tsujino ${ }^{5} \cdot$ Xiaochun Wang ${ }^{18}$
}

Published online: 13 May 2016

(C) Springer-Verlag Berlin Heidelberg 2016

\section{Erratum to: Clim Dyn \\ DOI 10.1007/s00382-016-2985-y}

In the original publication of the article, Fig. 11 was published incorrectly. The correct version of Fig. 11 is provided here.

The online version of the original article can be found under doi:10.1007/s00382-016-2985-y.

Matthieu Chevallier

matthieu.chevallier@meteo.fr

1 Centre National de Recherches Météorologiques (CNRM), Météo France/CNRS UMR3589, Toulouse, France

2 Recherche en Prévision Numérique Environnementale, Environnement et Changement Climatique Canada, Dorval, QC, Canada

3 Service Météorologique du Canada, Environnement et Changement Climatique Canada, Dorval, QC, Canada

4 Massachusetts Institute of Technology, Cambridge, MA, USA

5 Meteorological Research Institute (MRI), Japan Meteorological Agency, Tsukuba, Japan

6 Mercator Océan, Ramonville-Saint-Agne, France

7 Institut de Recherche pour le Développement (IRD), Toulouse, France

8 NOAA Geophysical Fluid Dynamics Laboratory (GFDL), Princeton, NJ, USA

9 Centre Européen de Recherche et de Formation Avancée au Calcul Scientifique (CERFACS), Toulouse, France
10 Met Office Hadley Centre, Exeter, UK

11 Euro-Mediterranean Centre for Climate Change, Bologna, Italy

12 National Centre for Earth Observation (NCEO), University of Reading, Reading, UK

13 Science Systems and Applications, Inc., Lanham, MD, USA

14 Global Modelling and Assimilation Office, NASA Goddard Space Flight Center (GSFC), Greenbelt, MD, USA

15 European Centre for Medium-Range Weather Forecasts (ECMWF), Reading, UK

16 Department of Earth Science Education, Kongju National University, Kongju, South Korea

17 National Institute for Geophysics and Volcanology, Bologna, Italy

18 Joint Institute for Regional Earth System Science and Engineering, UCLA, Los Angeles, CA, USA 

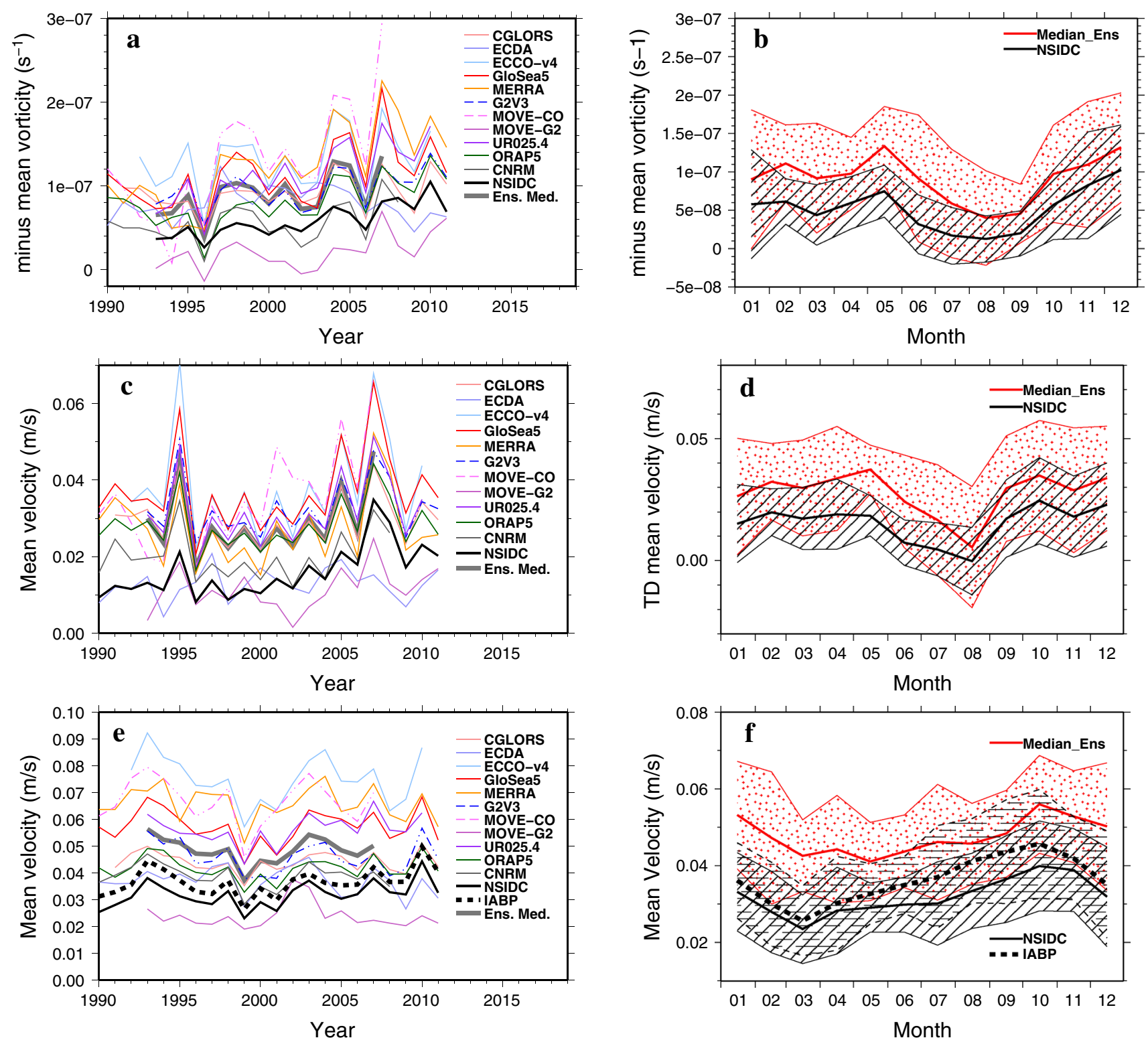

Fig. 11 a Annual vorticity (sign reversed, $\mathrm{s}^{-1}$ ) averaged over a sector (from $73^{\circ} \mathrm{N}$ to $82^{\circ} \mathrm{N}$ in latitude and from $175^{\circ} \mathrm{E}$ to $135^{\circ} \mathrm{W}$ in longitude) covering the Beaufort gyre for the different members of the ensemble, the median of the ensemble and the NSIDC product used as a reference. $\mathbf{b}$ Seasonal cycle for the mean vorticity for the median of the ensemble (thick red) and the NSIDC product (thick black) over the 1993-2007 period. Regions delimited by thin red and black lines and filled with red dots and black slanted patterns indicate the mean plus or minus one standard deviation for each month for the two

respective time series. $\mathbf{c}$ and $\mathbf{d}$ are, respectively, equivalent to $\mathbf{a}$ and $\mathbf{b}$ but for the mean speed $(\mathrm{m} / \mathrm{s})$ in the transpolar drift region tangent to the long axis of the box defined by Sumata et al. (2014). e and $\mathbf{f}$ are, respectively, equivalent to $\mathbf{c}$ and $\mathbf{d}$ but for the mean speed $(\mathrm{m} / \mathrm{s})$ calculated at the monthly location of the IABP buoys and also incorporate the IABP data (short thick dark dashes). The region of the mean plus or minus one standard deviation for IABP in (f) is delimited by thin dashed black lines and by a horizontal black pattern 